\title{
Los jagüeyes de los Montes de María en Sucre, Colombia: transformación alternativa y tradicional para acceso productivo y sobrevivencia
}

\author{
Cattle ponds of the mounts of Maria in Sucre, Colombia: alternative and \\ traditional transformation for productive access and survival
}

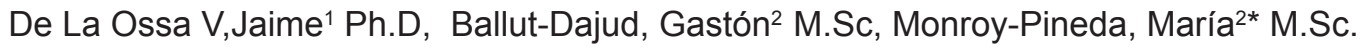

1 Universidad de Sucre, Facultad de Ciencias Agropecuarias, Grupo de Investigación en Biodiversidad Tropical, Colombia.

${ }^{2}$ Universidad de Sucre, Facultad de Ingeniería, Ingeniería Civil, Sucre, Colombia.

\section{Keywords:}

Cattle ponds; hydric resource; Montes de Maria; Sucre;

Colombia.

\section{Palabras Clave:}

Jagüeyes;

utilización;

recurso hídrico;

Montes de María;

Sucre, Colombia.

INFORMACIÓN

Recibido: 04-05-2016;

Aceptado: 27-11-2016.

Correspondencia autor:

mariacmonroy2007@hotmail.com

\section{Abstract}

The present study through the use of geographical information system, determines the cattle ponds presence in the municipal territories of Chalán, Colosó and Toluviejo, department of Sucre, Colombia. The study area formed part of Montes de María formation, identified as an area of tropical dry forest of the Colombian Caribbean. Were determined in total 500 units, of which the water mirror for municipality was: Chalan $0.129 \%$, Colosó $0.143 \%$ and Toluviejo $0.42 \%$, with a minimum range of area for unit between 0.1125 and 0.445 ha. The high dependence that the human populations have to satisfy the necessities of domestic water is demonstrated and it is compared with social variables such as: concentration of land tenure and poverty. To conclude, that the cattle ponds represents an important access system to the water of great value and importance for the study area.

\section{Resumen}

El presente trabajo a través del uso de herramientas de información geográfica, determina la presencia de jagüeyes en los territorios municipales de Chalán, Colosó y Toluviejo, departamento de Sucre, Colombia. La zona de estudio hace parte de la formación Montes de María, identificada como un área de bosque seco tropical del Caribe colombiano. Se determinaron en total 500 jagüeyes, de los cuales el espejo de agua por municipio fue: Chalan 0,129\%, Colosó 0,143\% y Toluviejo $0,42 \%$, con un rango mínimo de área por unidad entre 0,1125 y 0.445 ha. Se demuestra la alta dependencia que tienen las poblaciones humanas allí asentadas para satisfacer las necesidades de agua doméstica y se compara con variables sociales tales como: concentración de la tenencia de tierra y pobreza. Para concluir que los jagüeyes representan un sistema de acceso al agua de gran valor e importancia para la zona de estudio. 


\section{Introducción}

Según CERVANTES (1994) los jagüeyes son lagunas con aparente similitud con los lagos, su existencia puede corresponder a cualquier origen, drenaje y dimensiones. Igualmente, indica que permanecen relativamente estancados y son inestables, con variaciones en el nivel de agua permanentes, dependiendo del régimen pluvial.; pueden ser temporales o

DECLERK et al. (2006) indican que los jagüeyes juegan un importante rol en la conservación. CHARÁ et al. (2008) señalan que permiten, en efecto, el establecimiento y conservación de especies vegetales y organismos animales diversos, proveen alimento y refugio, tanto para seres vivos propios del agua como de aquellos que se asocian al sistema por las facilidades ofrecidas. Igualmente son fuente de agua para las comunidades rurales asentadas en su área de influencia (BOTERO et al., 2009; BALLUT-DAJUD y MONROY-PINEDA, 2015).

El bosque seco tropical que caracteriza a los Montes de María, según IAVH (1998), está definido como la formación vegetal que presenta una cobertura boscosa continua y que se distribuye entre los 0-1000 $\mathrm{m}$ de altitud, con temperatura superiores a los $24^{\circ} \mathrm{C}$, ubicado en el piso térmico cálido, con precipitaciones entre los 700 y $2.000 \mathrm{~mm}$ anuales; con uno o dos periodos marcados de sequía al año. De acuerdo con HERNÁNDEZ y SÁNCHEZ (1992) corresponde a los llamados bosques higrotropofíticos, bosque tropical caducifolio de diversos autores, bosque seco Tropical según Holdridge y al bosque tropical de baja altitud deciduo por sequía de la clasificación propuesta por la UNESCO.

En Colombia, se ha ido perdiendo vertiginosamente la cobertura de los bosques secos tropicales, si se asume que en tiempos coloniales ésta era cercana a la que naturalmente debería existir y mantenerse, su extensión total debió ser de unos $80.000 \mathrm{~km}^{2}$, o sea algo más del $7,3 \%$ del territorio nacional. La introducción al país de la raza bovina cebú, a finales del siglo XIX y comienzos del $X X$, que se concentró en los climas cálidos estacionales de sabana y en las zonas de bosque seco tropical de la región Caribe y de los valles interandinos, fue la principal causa de dicha transformación (OTERO et al., 2006).

Por otro lado, está la fragmentación, situación que es evidente para los Montes de María (GALVÁN-GUEVARA et al., 2015), y que como proceso dinámico reduce la continuidad de los hábitats a parches o islas de menor tamaño, más o menos conectadas entre sí en una matriz de hábitat diferentes al original (ARROYO-RODRÍGUEZ y MANDUJANO, 2009), con pérdida del paisaje original y sus recursos (DIDHAM, 1997; FAHRIG, 2003).
El presente trabajo evalúa la existencia de jagüeyes antrópicos en los tres principales municipios de los Montes de María en el departamento de Sucre, Colombia, al tiempo que discute las posibles razones de tipo ambiental que determinan su abundancia y su relación con el incremento de los procesos de transformación que vive la zona.

\section{Materiales y métodos}

Zona de estudio: Comprende el territorio de los municipios de Toluviejo, Colosó y Chalán, departamento de Sucre, Colombia. Forma parte de la porción sureste de los Montes de María (Figura 1). Se trabajó con un cubrimiento total de 50.129,3 ha.

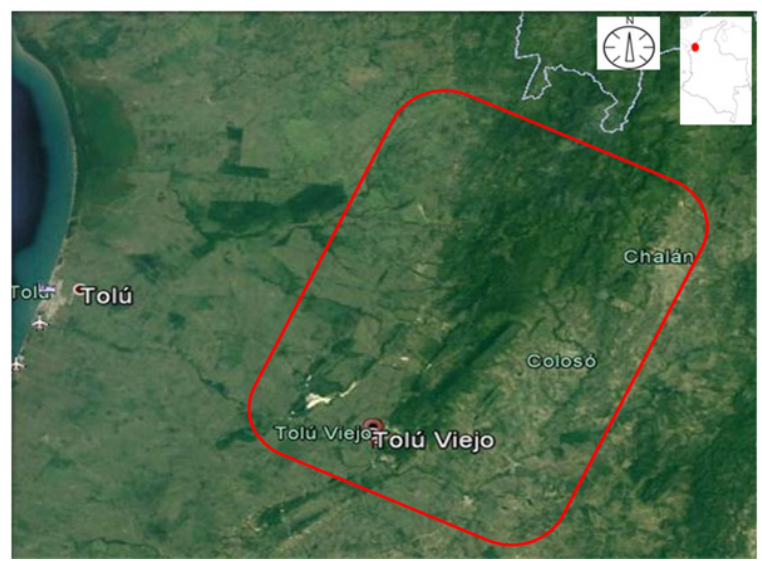

Figura 1. Zona de estudio. Google Earth versión libre.

Metodología: Para la construcción de los mapas que muestran la localización de los jagüeyes en los municipios de Toluviejo, Colosó y Chalan pertenecientes al departamento de Sucre, se utilizaron los software: Google Earth Pro y ArcGIS 9.3® licenciados a nombre de la Universidad de Sucre; atendiendo la siguiente metodología. En primera instancia, se descargaron del sitio web del Instituto Geográfico Agustín Codazzi, los archivos en forma de polígonos vectoriales de los municipios en mención, en formato shape (.shp). Seguido, se convirtieron en formato keyhole mark language $(. \mathrm{kml})$ para ser subidos al Google Earth y poder trabajar online, delimitando los jagüeyes dentro de los perímetros municipales.

Con una imagen reciente, (Octubre del 2016), se comenzó a delimitar uno a uno los jagüeyes haciendo un recorrido minucioso y detallado empleando un zoom que permitiera ampliar la zona de estudio con una altura del ojo o una altura de vuelo que iba desde los $50 \mathrm{~m}$ hasta los $1000 \mathrm{~m}$. Luego de digitalizar los jagüeyes, se exportaron a formato (.shp) para poder procesar la información y comenzar a elaborar la cartografía. 
Finalmente, se fueron elaborando uno a uno los mapas de los municipios, calculando las respectivas áreas en hectáreas y datos de estadística descriptiva.

\section{Resultados}

Los mapas de cada uno de los municipios en donde se evidencian los jagüeyes determinados para este trabajo, se presentan en la Figuras. 2, 3 y 4.

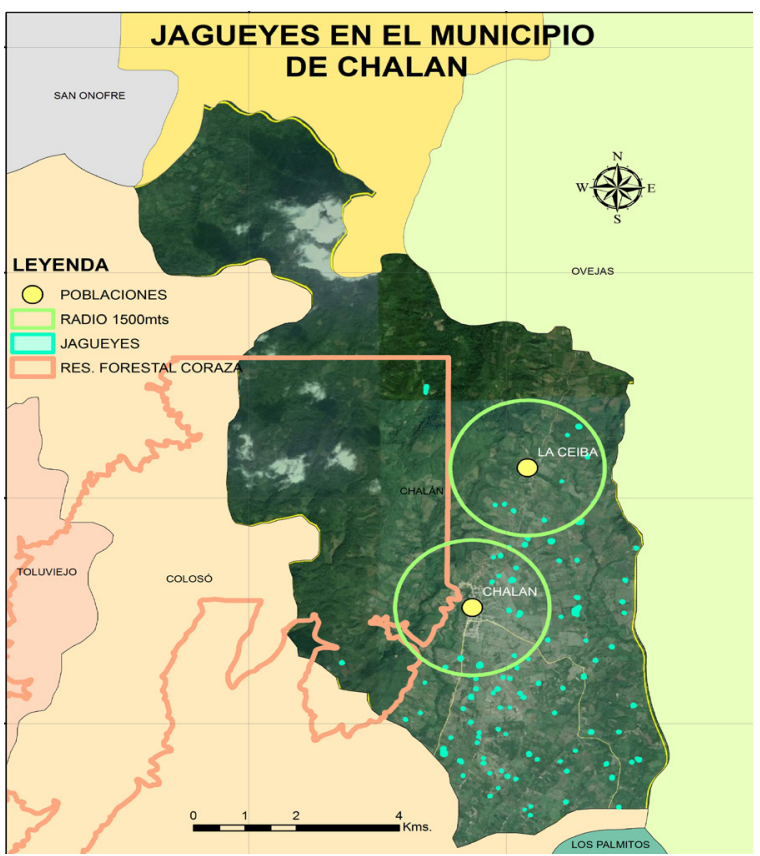

Figura 2. Mapa de los jagüeyes del municipio de Chalán, Sucre.

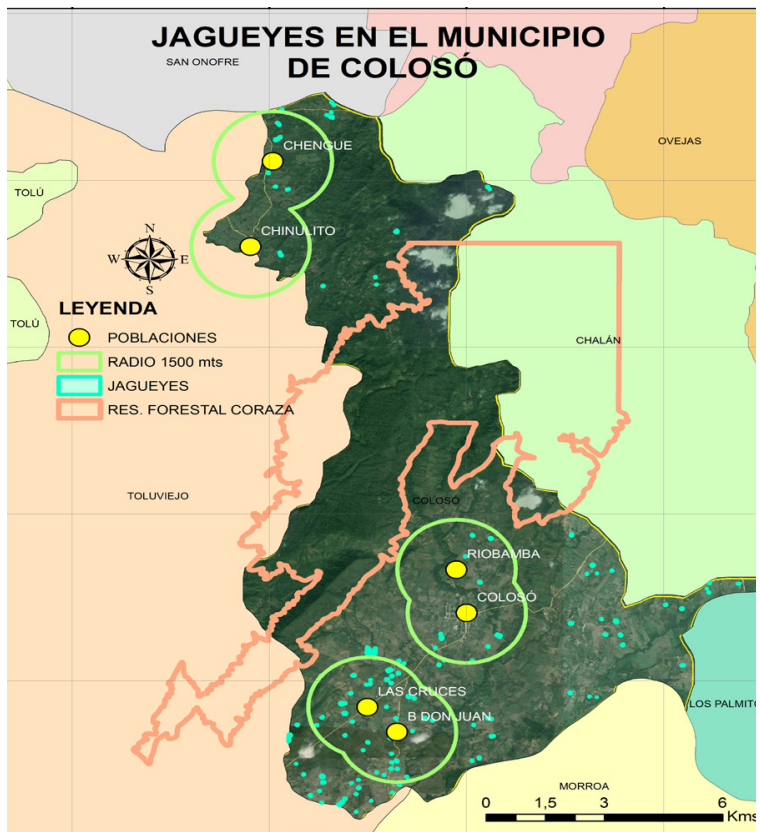

Figura 3. Mapa de los jagüeyes del municipio de Colosó, Sucre.

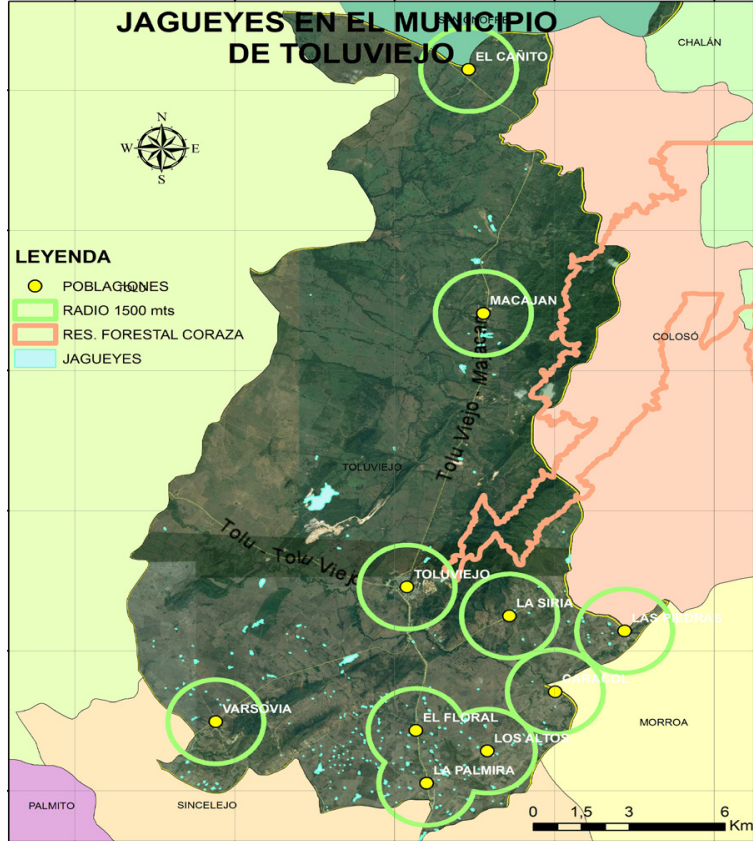

Figura 4. Mapa de los jagüeyes del municipio de Toluviejo, Sucre.

El área total muestreada para cada municipio, fue: Chalan 8.846,56 ha, Colosó 12721,22 ha y Toluviejo 28561,52 ha. Los resultados municipales referentes a número de jagüeyes, área minina, área máxima, área promedia y sus estadísticos no paramétricos se presentan en la Tabla 1. En total se total se registraron 500 unidades, con una participación por municipio de $20,4 \%, 25,6 \%$ y $54 \%$, respectivamente.

En cuanto al número de jagüeyes por unidad de área, se tiene: Chalan 1 por cada 78,98 ha, Colosó 1 por cada 99,38 ha y para Toluviejo 1 por cada 105,78 ha. En total para el área de estudio se tendría 1 jagüey por cada 110,2 ha. El espejo de agua aportado por los jagüeyes representa para cada uno de los territorios municipales: Chalan $0,129 \%$, Colosó $0,143 \%$ y Toluviejo $0,42 \%$.

En la Tabla 3 se muestra la distribución concentrada de los jagüeyes tomando un radio de $1.500 \mathrm{~m}$ desde el centro poblado más cercano en cada municipio estudiado.

\section{Discusión}

Según BALLUT-DAJUD y MONROY-PINEDA (2015) en el municipio de Sincelejo, departamento de Sucre, Colombia, la representación de los jagüeyes corresponde al $0,43 \%$ del territorio municipal, situación que es compartida en este estudio con el municipio de Toluviejo y que es porcentualmente mayor a lo encontrado para los otros dos municipios analizados. 
Tabla 1: Jagüeyes registrados para los municipio de este estudio (ha).

\begin{tabular}{cccccccc}
\hline Municipio & $\mathbf{N}^{\circ}$ jagüeyes & Área mínima & Área máxima & Área promedio & Sumatoria total & Desviación estándar & Varianza \\
\hline Chalan & 102 & 0,00785 & 1,1305 & 0,1125 & 11,48 & 0,1565 \\
Colosó & 128 & 0,0076 & 4,01 & 0,143 & 18,31 & 0,0245 \\
Toluviejo & 270 & 0,00154 & 39,99 & 0,445 & 120,01 & 2,1288 \\
\hline
\end{tabular}

Tabla 3. Concentración de los jagüeyes entorno a lugares poblados dentro de cada área municipal (1.500 m de radio, área en ha).

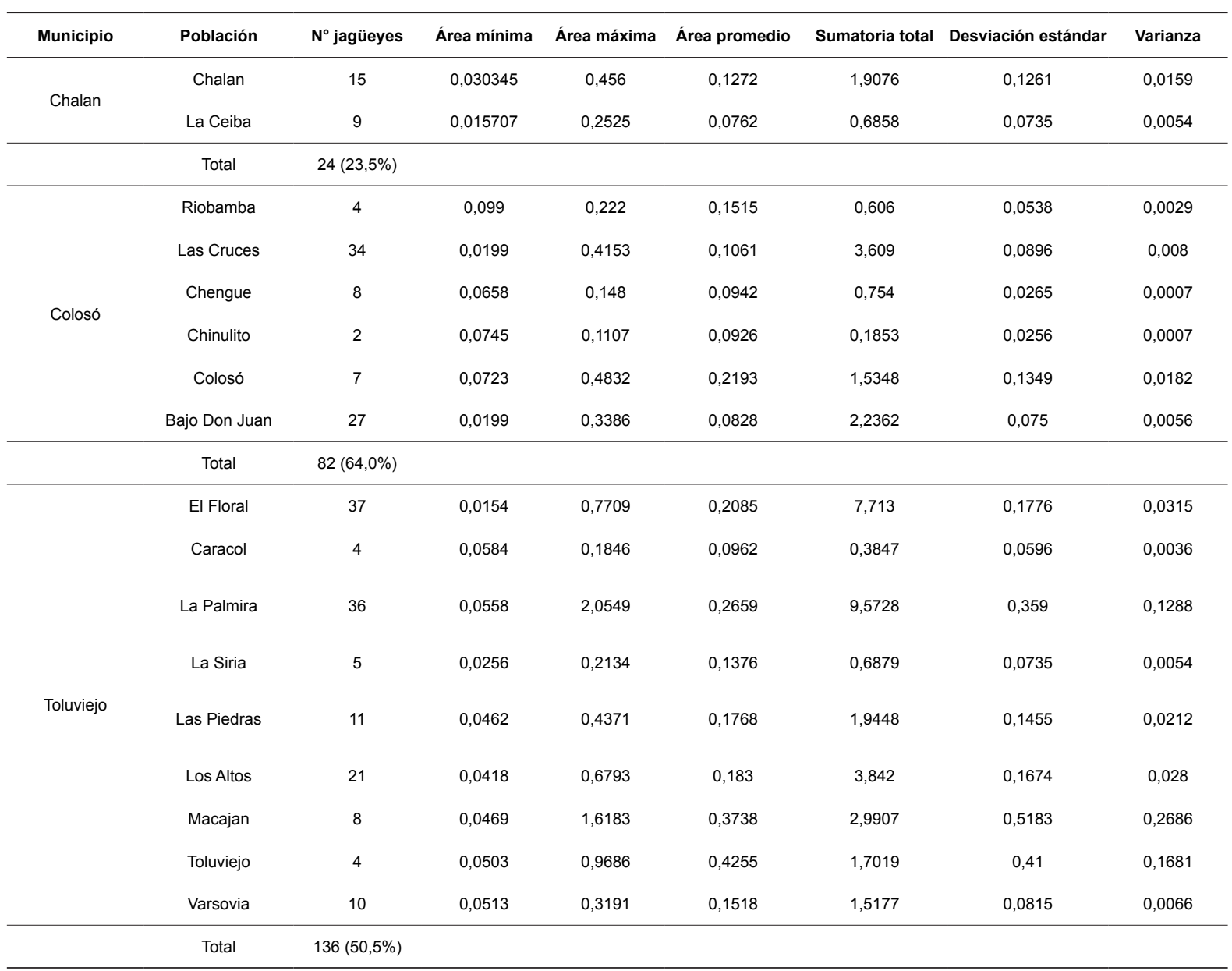

En cuanto al promedio de un jagüey por cada 95 ha (BALLUT-DAJUD y MONROY-PINEDA (2015), información que es menor de lo hallado para el municipio de Chalan ( 1 por cada 78,98 ha) y es menor que lo registrado para el municipio de Toluviejo (1 por cada 105,78 ha) y en términos generales es similar a lo hallado para el municipio de Colosó (1 por cada 99,38 ha). En cuanto al rango de espejo de agua que poseen los jagüeyes, se tiene coincidencia con lo establecido para el municipio de Sincelejo por BALLUT-DAJUD y MONROY-PINEDA (2015).

El registro de un área promedio entre 0,1125 y 0.445 ha, es decir un espejo de agua menor a una hectárea coincide con lo hallado por BALLUT-DAJUD y MONROYPINEDA (2015) para el municipio de Sincelejo, y coincide con los expresado por BOTERO et al. (2009), quienes señalan que se trata de unidades cuya utilización estaría en concordancia con el manejo ganadero en pequeña escala o asociado a trashumancia, práctica que es común y tradicional en la zona de estudio. No obstante, la baja representatividad que a nivel de espejo de agua tienen estas lagunas antrópicas en relación con el área total municipal permite determinar que en la época la sequía es acentuada en el bosque seco tropical (IAVH, 1998; OTERO et al., 2006), un alto déficit de agua para consumo humano y producción pecuaria impone seria restricciones de sobrevivencia. 
El hallazgo de la concentración de jagüeyes respecto de sitios habitación humana, en un radio de 1.500 m y que representa $23,5 \%$ para Chalan, $64,0 \%$ para Colosó y $50,3 \%$ para Toluviejo, muestra en general que el uso de estas unidades de almacenamiento de agua tiene un uso significativo para consumo humano. Por otro lado, aquellos jagüeyes que son parte de las empresas ganaderas, como lo señalan BOTERO et al. (2009) también son usados por los habitantes aledaños, lo que según los autores mencionados, ha venido suscitando conflictos sociales, por un lado los propietarios manifiestan la necesidad y exclusividad en el uso del recurso, que en su caso es una fuerte limitante para la producción, mientras que los habitantes circundantes, asentados en poblados carentes de acueductos, muchas veces con altos índices de NBI, (Necesidades Básicas Insatisfechas) deben por fuerza mayor acceder al recurso para obtener agua para todos los usos domésticos.

Lo anterior, de acuerdo con los datos poblacionales de cada municipio analizado, y en el cual la población rural es: Chalan 38,1\%, Colosó 49,3\% y Toluviejo 71\%, además de la deficiencia de los acueductos urbanos y la inexistencia de los mismos para el área rural (DNP, 2011; MUNICIPIO DE COLOSÓ, 2012; MUNICIPIO DE TOLUVIEJO, 2012), explicaría la dependencia de los jagüeyes y la necesidad de los mismos en la satisfacción de la necesidades hídricas de la zona de estudio.

La concentración de los jagüeyes entorno a poblaciones humanas también es un indicador de la concentración de la tierra que existe en la zona de estudio. MENCO (2012) señalan un Índice de Gini para Chalán de 0,69, para Colosó de 0,76 y para Toluviejo de 0,84, que demuestra que la tierra está en manos de unos pocos habitantes. Por otro lado, se puede corroborar esta apreciación mediante el Índice de Pobreza Multidimensional, que según AGUILERA-DIAZ (2013), muestra que el $92 \%$ de la población rural de la subregión está bajo condiciones de pobreza, encontrándose que entre los factores que más inciden, además del empleo formal, los logros educativos y condiciones de la vivienda, está el acceso a los servicios públicos domiciliarios, de los cuales el acceso al agua potable hace un gran peso y que como se ve en este estudio es aportado en una buena parte por los jagüeyes. Pertinentemente, señala que el porcentaje de la población en situación de miseria es de 37,4 para Chalan, 30,6 para Colosó y 17,9 para Toluviejo, lo que escenifica la grave situación social (MENCO, 2009) y la dependencia que tienen los habitantes de la zona de estudio de los jagüeyes para uso doméstico.

\section{Conclusiones}

Tomando en cuenta la importancia multifuncional que los jagüeyes poseen, tanto en lo ecológico como en lo social y en lo económico, su conservación e incremento así como su interconexión es una necesidad, en especial para una zona como los Montes de María, en donde la matriz de bosque seco tropical impone condiciones restrictivas una buena parte del año y en donde la situación de pobreza es notoria y el acceso al agua es una limitante de productividad y sobrevivencia.

Dado el valor regional de los jagüeyes, existe la necesidad de profundizar en su estudio, aspectos productivos, conservacionistas y sociales deben hacer parte del trabajo científico requerido, las evidencias aquí plasmadas indican que existe un amplio campo para la investigación, que no admite, dadas las circunstancias sociales de la zona una postergación, menos aún la espera de soluciones efectivas como objetivo primordial.

\section{Referencias}

AGUILERA-DÍAZ, M. 2013. Montes de María: Una subregión de economía campesina y empresarial. Documento de trabajo sobre economía regional. Banco de la República. Cartagena, Colombia.

ARROYO-RODRIGUEZ, V.; MANDUJANO, S.. 2009. Conceptualization and measurement of habitat fragmentation from the primates' perspective. International Journal of Primatology 30: 497-514.

BALLUT-DAJUD, G.; MONROY-PINEDA, M.C. 2015. Los jagüeyes del municipio de Sincelejo, Sucre, Colombia. Rev Colombiana Cienc Anim. 7 (1):80-83.

BOTERO, A.L.; DE LA OSSA, V.J.; ESPITIA, P.A.; DE LA OSSA-LACAYO, A. 2009. Importancia de los jagüeyes en las sabanas del Caribe colombiano. Rev. col. Cien. Anim. 1 (1):71-84.

CERVANTES, M. 1994 (Compilador). Guía regional para el conocimiento, manejo y utilización de los humedales del noroeste de México. ITESM-Campus Guaymas/CECARENA, México: 27-82. 
CHARÁ, J.; PEDRAZA, O.G.; GIRALDO, S.L. 2008. Ganadería del futuro: Investigación para el desarrollo. Corredores ribereños como herramienta de protección de ambientes acuáticos en zonas ganaderas, Cap. 5. CIPAV, Cali, Colombia: 111-129.

DECLERCK, S.; DE BIE, T.; ERCKEN, D.; HAMPEL, H.; SCHRIJVERS, S.; VAN WICHELEN, J.; GILLARD, V.; MANDIKI, R.; LOSSON, B.; BAUWENS, D.; KEIJERS, S.; VYVERMAN, W.; GODDEERIS, B.; DE MEESTER, L.; BRENDONCK, L.; MARTENS, K. 2006. Ecological characteristics of small farmland ponds: Associations with land use practices at multiple spatial scales. Biological Conservation 131 (4):523-532.

DIDHAM, R.K. 1997. The influence of edge effects and forest fragmentation on leaf litter invertebrates in Central Amazonia. Págs. 55-70. En: Laurance, W.F.; Bierregaard, R.O. Jr. (Eds.). Tropical forest remnants: ecology, management and conservation of fragmented communities. The University Chicago Press, Chicago, London.

DNP - Departamento Nacional de Planeación. 2011. Ficha Municipal. Alcaldía Municipal de Chalán. Disponible en: XXXXXX. Consultado: 10-08-2016.

FAHRIG, L. 2003. Effects of habitat fragmentation on biodiversity. Annual Review of Ecology Evolution and Systematics 34: 487-515.

GALVÁN-GUEVARA, S.; BALLUT-DAJUD, D.; DE LA OSSAV,J. 2015. Determinación de la fragmentación del bosque seco del arroyo Pechelín, Montes de María, Caribe, Colombia. Biota Colombiana 16 (2):121-129.

HERNÁNDEZ-CAMACHO, J.; SÁNCHEZ, H. 1992. Biomas terrestres de Colombia. Págs. 153-173 En: Halffter, G (ed.). La diversidad biológica iberoamericana I. Acta Zoológica Mexicana, México. 390 pp.

IAVH. 1998. Informe Nacional sobre el Estado de la Biodiversidad. En: Chaves, M.E. Arango, N. (Eds.). Tomo I: Diversidad Biológica. Bogotá.

MENCO. D. 2009. Desarrollo rural y problema de tierras en los Montes de María. Limitaciones y perspectivas. Revista Desarrollo Local Sostenible (DELOS) 2 (6). Disponible en: www.eumed.net/rev/delos/06. Consultado: 09-07-2015.

MENCO, D. 2012, Tenencia y uso de la tierra rural en los Montes de María. Consultor para Bancos de Proyectos de la Fundación MSI Colombia en el Programa Cimientos, para Montes de María. (Mimeo).

MUNICIPIO DE COLOSÓ. 2012. Plan de desarrollo. 2012-2015. Municipio de Colosó. Disponible en: http://www. coloso-sucre.gov.co/apc-aa-files/39336265383238663436386532613634/pdm-coloso-2012-2015-la-gerencia-delas-cosas-grandes 4.pdf. Consultado: 10-08-2016.

MUNICIPIO DE TOLUVIEJO. 2012. Plan de desarrollo. 2012-2015. Municipio de Colosó. Disponible en: http:// www.toluviejo-sucre.gov.co/apc-aa-files/63616163626565386437343032343466/plan de desarrollo toluviejo sucre 012 2015.pdf. Consultado: 10-08-2016.

OTERO, E.; MOSQUERA, L.; SILVA, G.; GUZMÁN, J. 2006. Bosque seco tropical en Colombia. Libros de la Colección Ecológica del Banco de Occidente. Cali, Colombia. 